\title{
Questes
}

\section{La faim et l'appétit : éléments bibliographiques}

\section{Anne-Laure Lallouette}

\section{(2) OpenEdition}

\section{Journals}

\section{Édition électronique}

URL : http://journals.openedition.org/questes/2759

DOI : 10.4000/questes.2759

ISSN : 2109-9472

\section{Éditeur}

Les Amis de Questes

\section{Édition imprimée}

Date de publication : 15 juin 2007

Pagination : 112-117

ISSN : 2102-7188

\section{Référence électronique}

Anne-Laure Lallouette, «La faim et l'appétit : éléments bibliographiques », Questes [En ligne], 12 | 2007. mis en ligne le 15 janvier 2014, consulté le 15 septembre 2020. URL : http://journals.openedition.org/ questes/2759 


\section{Bibliographie}

\section{Anne-Laure LALLOUETTE}

\section{I- $\quad$ La faim et l'alimentation, réelle ou symbolique}

ALEXANDRE-BIDON Danièle, Une archéologie du goût: céramique et consommation. Moyen Âge - Temps modernes, Paris, Picard, 2005.

Banquets et manières de table au Moyen Âge, Aix-en-Povence, CUER MA (coll. Senefiance, 38), 1996.

BITSCH Irmgard, EHLERT Trude et ERTZDORFF Xenja (von) (éd.), Essen und Trinken in Mittelalter und Neuzeit, Actes du colloque du 10 au 13 juin 1987, Sigmaringen, J. Thorbecke, 1987.

BOBER Phyllis Pray, Art, Culture and Cuisine: Ancient and Medieval Gastronomy, Chicago et London, University of Chicago Press, 1999.

BRUEGEL Martin et LAURIOUX Bruno, Histoire et identités alimentaires en Europe, Paris, Hachette, 2002.

DESPORTES Françoise, Le Pain au Moyen Âge, Paris, Olivier Orban, 1987.

FARRIER Susan E., "Hungry Hereos in Medieval Litareture », in Weiss Adamson MELITTA (éd.), Food in the Middle Ages. A Book of Essays, New York et London, Garland, 1995, p. 145-160.

GALLOUIN François, Évolution historique des concepts de faim, satiété, appétits, Thèse de doctorat sous la direction de Jacques Roger, Université de Paris I Panthéon-Sorbonne, 1988.

GLIXELLI Stefan, «Les contenances de la table », Romania, XLVII (1921), p. $1-40$.

GLÉNISSON Jean, « Une administration médiévale aux prises avec la disette. La question des blés dans les provinces italiennes de l'État pontifical en 1374$1375 »$, Le Moyen Âge, 3-4 (1951), p. 303-306. 
GUERREAU-JALABERT Anita, " Aliments symboliques et symbolique de la table dans les romans arthuriens (XII ${ }^{\mathrm{e}}-\mathrm{XIII}^{\mathrm{e}}$ siècles) », dans Annales E.S.C., 3 (1992), p. 561-594.

HENISCH Bridget Ann, Fast and Feast. Food in Medieval Society, London, University of Pennsylvania Press, 1978.

LAMBERT Carole (dir.), Du manuscrit à la table. Essais sur la cuisine au Moyen Âge et répertoire des manuscrits médiévaux contenant des recettes culinaires, Paris, Champion, 1992.

LAURIOUX Bruno, Le Moyen Âge à table, Paris, A. Biro, 1989.

- «La cuisine des médecins à la fin du Moyen Âge», dans Maladie, Médecines et Sociétés. Approches historiques pour le présent, Paris, 1993, t. II, p. 136-148.

- Le règne de Taillevent. Livres et pratiques culinaires à la fin du Moyen Âge, Paris, Publications de la Sorbonne, 1997.

- Manger au Moyen Âge. Pratiques et discours alimentaires en Europe aux XIV et $X V^{e}$ siècles, Paris, Hachette, 2002.

- «Les voyageurs et la gastronomie en Europe au XV $\mathrm{XV}^{\mathrm{e}}$ siècle », dans Le désir et le goût. Une autre histoire : XIII $-X V I I I^{e}$ siècles, Odile REDON, Line TEISSEYRE-SALLMANN et Sylvie STEINBERG (éd.), Actes du colloque international à la mémoire de Jean-Louis Flandrin, Saint-Denis, Septembre 2003, Saint-Denis, Presses Universitaires de Vincennes, 2005, p. 99-117.

- Une histoire culinaire du Moyen Age, Paris, Champion, 2005.

Manger et boire au Moyen Âge, Actes du colloque de la Faculté des Lettres de Nice, 15-17 octobre 1982, Paris, Les Belles Lettres, 1984.

MONTANARI Massimo, Alimentazione e cultura nel Medioevo, Bari, Laterza, 1988.

- La faim et l'abondance : histoire de l'alimentation en Europe, Paris, Seuil, 1995.

MÜHLETHALER Jean-Claude, « De la frugalité de l'ermite au faste du prince : les codes alimentaires dans la littérature médiévale », dans Manger, Cours général public 1995-1996, Lausanne, Payot (coll. Publications de 1'Université de Lausanne, LXXXIX), 1996, p. 7-35.

NICOUD Marilyn, "Les pratiques diététiques à la cour de Francesco Sforza ", in Scrivere il Medioevo. Lo spazio, la santità, il cibo, Bruno LAURIOUX et Laurence MouliniER-Brogi (éd.), Roma, Viella, 2001, p. 393-405. 
Plaisirs et manières de table aux XIV et $X V^{e}$ siècles, catalogue de l'exposition du 23 avril au 29 juin 1992, Musée des Augustins, Toulouse, Musée des Augustins, 1992.

POLET Caroline et ORBAN Rosine, Les dents et les ossements humains. Que mangeait-on au Moyen Âge?, dans Typologie des sources du Moyen Âge occidental, Léopold GÉNICOT (dir.), Turnhout, Brepols, 2001.

REDON Odile et LAURIOUX Bruno, « Histoire de l'alimentation entre Moyen Âge et Temps moderne ", in Le désir et le goût. Une autre histoire : XIII ${ }^{e}-X V I I I^{e}$ siècles, Odile REDON, Line TEISSEYRE-SALLMANN et Sylvie STEINBERG (éd.), Actes du colloque international à la mémoire de Jean-Louis Flandrin, Saint-Denis, septembre 2003, Saint-Denis, Presses Universitaires de Vincennes, 2005, p. 53-96.

ROSENBERGER Bernard, "Diététique et cuisine dans l'Espagne musulmane $\mathrm{du} \mathrm{XIII}^{\mathrm{e}}$ siècle », dans Le désir et le goût. Une autre histoire : XIII ${ }^{e-} X V I I I^{e}$ siècles, Odile Redon, Line Teisseyre-Sallmann et Sylvie Steinberg (éd.), Actes du colloque international à la mémoire de Jean-Louis Flandrin, SaintDenis, Septembre 2003, Saint-Denis, Presses Universitaires de Vincennes, 2005, p. 175-191.

WEISS ADAMSON Melitta (éd.), Food in the Middle Ages. A Book of Essays, New York et London, Garland, 1995.

WILSON Anne C., The Appetite and the Eye: Visual Aspects of Food and its Presentation within their historic Context, Edinburgh, Edinburgh University Press, 1991.

\section{II- La faim et l'Église : de la gourmandise au jeûne}

BAUER Gerd, «In Teufels Küche », dans Essen und Trinken in Mittelalter und Neuzeit, Irmgard BITSCH, Trude EHLERT et Xenja von ERTZDORFF (éd.), Sigmaringen, Thorbecke, 1987, p. 127-142.

BELL Rudolph M., L'anorexie sainte. Jeûne et mysticisme du Moyen Âge à nos jours, Paris, PUF, 1994.

BYNUM Caroline, Jeûnes et festins sacrés. Les femmes et la nourriture dans la spiritualité médiévale, Paris, Éditions du Cerf, 1994. 
CASAGRANDE Carla et VECCHIO Silvana, Histoire des péchés capitaux au Moyen Âge, Paris, Aubier, 2000.

CASTRO Teresa (de), «El gusto alimentario en la doctrina moral de la iglesia en la baja Edad Medi según Hernando de Talavera», dans Micrologus : Natura, scienze e società medievali, 10 (2002), p. 379-399.

DELUMEAU Jean, Le péché et la peur. La culpabilisation en Occident : XIII ${ }^{e}-$ XVIII siècles, Paris, Fayard, 1994.

FRAISE Nathalie, L'anorexie mentale et le jeûne mystique de Moyen Âge : faim, foi et pouvoir, Paris et Montréal, L’Harmattan, 2000.

GRANDPERRIN Nathalie, La loi et la faute: interdits et transgression dans la littérature arthurienne des XII et XIII ${ }^{e}$ siècles, Thèse de doctorat, Littérature et civilisations françaises : Moyen Âge et Renaissance, Montpellier III, 1996.

GWARA SCOTT James, « Gluttony, Lust and Penance in the B-Text of Aislinge Meic Conglinne », Celtica, 20 (1988), p. 53-72.

LOTTIN D. Odon, «La doctrine morale des mouvements premiers de l'appétit sensitif », dans Archives d'histoire doctrinale et littéraire du Moyen Âge, 1931, Paris, Vrin, 1932, p. 49-173.

MILLER William Ian, « Gluttony », Representations, 60 (1997), p. 92-112.

MOSES David, «Soul Food and Eating Habits: What's at Steak in a Medieval Monk's Diet ? », Medium AEvum, 75, 1 (2006), p. 213-222.

N'DIAYE Catherine, La gourmandise : délices d'un péché, Paris, Autrement, 1996.

ROGUET Yves, "Gloutonnerie, gourmandise et caquets ", dans Banquets et manières de table au Moyen Âge, Aix-en-Provence, CUER MA, 1996, p. 255277.

SUAREZ-NANI Tiziana, «Du goût et de la gourmandise selon Thomas d'Aquin ", dans Micrologus : Natura, scienze e società medievali, 10 (2002), p. 313-334.

TRONE Georde Andrew, "The Cry of Dereliction in Purgatio XXIII », Dante Studies, 113 (1995), p. 111-129.

VOGEL Cyrille (éd. et trad.), Le pécheur et la pénitence au Moyen Âge, Paris, Éditions du Cerf, 1969. 
VOGÜÉ Adalbert (de), «Travail et alimentation dans les règles de saint Benoît et du maître », Revue bénédictine, 74 (1964), p. 242-251.

\section{III- Les appétits sexuels}

BALADIER Charles, Erôs au Moyen Âge : amour, désir et délectation morose, Paris, Éditions du Cerf, 1999.

CAMILLE Michael, L'art de l'amour au Moyen Âge : objets et sujets de désir, Cologne, Könemann, 2000.

GILLON Louis-Bertrand, La théorie des oppositions et la théologie du péché au XIII ${ }^{e}$ siècle, Paris, Vrin, 1938.

HERGEMÖLLER Bernd-Ulrich, Krötenkuss und schwarzer Kater : Ketzerei, Götzendienst und Unzucht in der inquisitorischen Phantasie des 13. Jahrhunderts, Warendorf, Fahlsbuch, 1996.

\section{IV- Les autres appétits et la soif}

DUFOURNET Jean, "Variations sur un motif. La taverne dans le théâtre arrageois du XIII ${ }^{\mathrm{e}}$ siècle ", dans Hommage à Jean-Charles Payen. Farai chansoneta novele, Caen, Centre de publications de l'Université de Caen, 1989, p. 161-174.

La Gastronomie au Moyen Age : 150 recettes de France et d'Italie, textes traduits et présentés par Odile REDON, Françoise SABBAN et Silvano SERVENTI, Paris, Stock, 1993.

HERBERT McAVOY Liz et WALTERS Teresa, Consuming Narratives: Gender and Monstruous Appetite in the Middle Ages and the Renaissance, Cardiff, University of Wales, 2002.

LAFORTUNE-MARTEL Agathe, Fête noble en Bourgogne au XV siècle. Le banquet du Faisan (1454), Montréal et Paris, Bellarmin et Vrin, 1984. 
OPSOMER-HALLEUX Carmélia, L'art de vivre en santé : images et recettes du Moyen Age: le Tacuinum Sanitatis, manuscrit 1041 de la bibliothèque de l'université de Liège, Liège, Éditions du Perron, 1991.

REYNOLDS PHILIP Lyndon, Food and the Body: some Peculiar Questions in High Medieval Theology, Leiden et Boston, Brill, 1999.

STUIP René Ernst Victor, "Je meurs de soif et suy a la fontaine ", dans Mélanges de linguistique et de littérature offerts à Léin Geschiere, Antonij DEES, Jean-Robert SMEETS, René Ernst Victor STUIP (éd.), Amsterdam, Rodopi, 1975.

VITZ Evelyn Birge, Medieval Narrative and Modern Narratology: Subjects and Objects of Desire, New York et London, New York University Press, 1989. 